\title{
A MODERN VIEW OF WIDE-ANGLE EXCLUSIVE SCATTERING
}

\author{
P. KROLL \\ Fachbereich Physik, Universität Wuppertal, \\ D-42097 Wuppertal, Germany \\ Email:kroll@physik.uni-wuppertal.de
}

\begin{abstract}
The basic theoretical ideas of the handbag mechanism for wide-angle exclusive scattering reactions are discussed and, with regard to the present experimental program carried out at JLab, its application to Compton scattering is reviewed in some detail. Results for other wide-angle reactions such as two-photon annihilations into pairs of hadrons or virtual Compton scattering are presented as well.

Invited talk presented at the $\mathrm{N}^{*} 2002$ workshop on the physics of excited nucleons, Pittsburgh, October 2002
\end{abstract}

\section{Introduction}

Recently a new approach to hard Compton scattering (CS) off protons has been proposed where the process amplitudes factorize into a hard partonlevel subprocess, Compton scattering off quarks, and generalized parton distributions (GPDs) which encode the soft physics (see Fig. 1). This so-called handbag mechanism applies to deep virtual Compton scattering (DVCS) ${ }^{1}$ characterized by a large virtuality, $Q^{2}$, of the incoming photon and a small squared invariant momentum transfer, $-t$, from the incoming to the outgoing proton $\left(-t / Q^{2} \ll 1\right)$. Subsequently it has been realized that the handbag mechanism also applies to wide-angle CS ${ }^{2,3}$ for which $-t$ (and $-u$ ) are large but the photon virtuality is small or even zero $\left(-Q^{2} / t \ll 1\right)$. It is believed now that the handbag mechanism is the relevant physics for a large number of deep virtual and wide-angle exclusive reactions such as electroproduction of mesons or two-photon annihilations into pairs of hadrons.

Wide-angle exclusive reactions and in particular real CS are the subject of my talk. The handbag mechanism in CS is described in Sect. 2. The large $-t$ behaviour of GPDs and form factors is discussed in Sect. 3 and predictions for CS are given. Characteristic results for other wide-angle exclusive processes are presented in Sect. 4. 

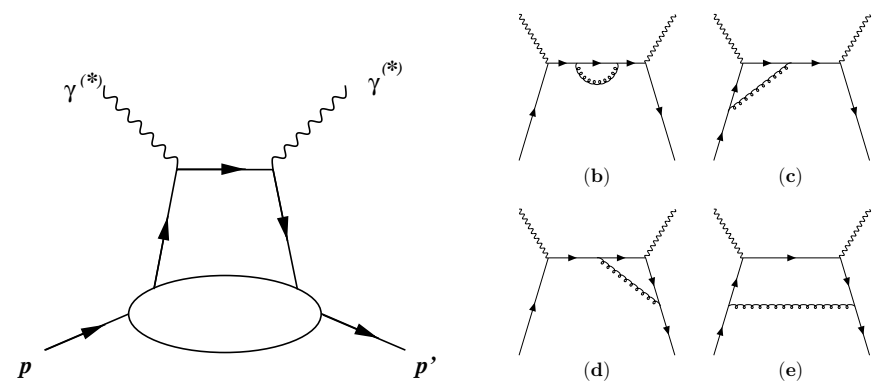

(b)

(c)

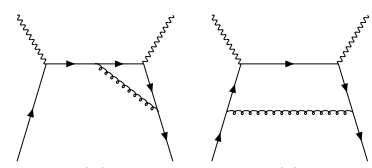

(d)

(e)

Figure 1. Handbag diagram for Compton scattering (left) and sample NLO pQCD Feynman graphs for its parton subprocess $\gamma q \rightarrow \gamma q$.

\section{Wide-angle Compton scattering}

For Mandelstam variables $s,-t$ and $-u$ that are large as compared to a typical hadronic scale $\Lambda^{2}$ where $\Lambda$ being of order $1 \mathrm{GeV}$, it can be shown that the handbag diagram shown in Fig. 1 describes CS. To see this it is of advantage to work in a symmetrical frame which is a c.m.s rotated in such a way that the momenta of the incoming $(p)$ and outgoing $\left(p^{\prime}\right)$ proton momenta have the same light-cone plus components. In this frame the skewness, defined as

$$
\xi=\frac{\left(p-p^{\prime}\right)^{+}}{\left(p+p^{\prime}\right)^{+}}
$$

is zero. The bubble in the handbag is viewed as a sum over all possible parton configurations as in deep ineleastic lepton-proton scattering (DIS). The crucial assumptions in the handbag approach are that of restricted parton virtualities, $k_{i}^{2}<\Lambda^{2}$, and of intrinsic transverse parton momenta, $\mathbf{k}_{\perp \mathbf{i}}$, defined with respect to their parent hadron's momentum, which satisfy $k_{\perp i}^{2} / x_{i}<\Lambda^{2}$, where $x_{i}$ is the momentum fraction parton $i$ carries.

One can then show ${ }^{3}$ that the subprocess Mandelstam variables $\hat{s}$ and $\hat{u}$ are the same as the ones for the full process, Compton scattering off protons, up to corrections of order $\Lambda^{2} / t$ :

$$
\hat{s}=\left(k_{j}+q\right)^{2} \simeq(p+q)^{2}=s, \quad \hat{u}=\left(k_{j}-q^{\prime}\right)^{2} \simeq\left(p-q^{\prime}\right)^{2}=u .
$$

The active partons, i.e. the ones to which the photons couple, are approximately on-shell, move collinear with their parent hadrons and carry a momentum fraction close to unity, $x_{j}, x_{j}^{\prime} \simeq 1$. Thus, like in DVCS, the physical situation is that of a hard parton-level subprocess, $\gamma q \rightarrow \gamma q$, and a soft emission and reabsorption of quarks from the proton. The light-cone 
helicity amplitudes ${ }^{4}$ for wide-angle CS then read

$$
\begin{aligned}
M_{\mu^{\prime}+, \mu+}(s, t)=\frac{e}{2}[ & T_{\mu^{\prime}+, \mu+}(s, t)\left(R_{V}(t)+R_{A}(t)\right) \\
& \left.+T_{\mu^{\prime}-, \mu-}(s, t)\left(R_{V}(t)-R_{A}(t)\right)\right], \\
M_{\mu^{\prime}-, \mu+}(s, t)=- & \frac{e}{2} \frac{\sqrt{-t}}{2 m}\left[T_{\mu^{\prime}+, \mu+}(s, t)+T_{\mu^{\prime}-, \mu-}(s, t)\right] R_{T}(t) .
\end{aligned}
$$

$\mu, \mu^{\prime}$ denote the helicities of the incoming and outgoing photons, respectively. The helicities of the protons in $M$ and of the quarks in the hard scattering amplitude $T$ are labeled by their signs. $m$ denotes the mass of the proton. The hard scattering has been calculated to next-to-leading order perturbative $\mathrm{QCD}^{5}$, see Fig. 1 . To this order the gluonic subprocess, $\gamma g \rightarrow \gamma g$, has to be taken into account as well. The form factors $R_{i}$ represent $1 / \bar{x}$-moments of GPDs at zero skewness. This representation which requires the dominance of the plus components of the proton matrix elements, is a non-trivial feature given that, in contrast to DIS and DVCS, not only the plus components of the proton momenta but also their minus and transverse components are large here. It is interesting to note that the DVCS amplitudes ${ }^{1}$

$$
\begin{aligned}
M^{\mathrm{DVCS}} \propto & \bar{u}\left(p^{\prime}\right) \gamma^{+} u(p) \int_{-1}^{1} d \bar{x} H(\bar{x}, \xi, t)\left[\frac{1}{\bar{x}-\xi+i \epsilon}+\frac{1}{\bar{x}+\xi-i \epsilon}\right] \\
& +E, \widetilde{H}, \widetilde{E}-\text { terms }
\end{aligned}
$$

although being derived for large $Q^{2}$ and small $-t$, embodies the wide-angle amplitudes (3) as can easily been seen by setting $\xi=0$ and evaluating the kinematical factors in front of the integral at large $-t$. The integrals over $H, E$ and $\widetilde{H}$ turn into the form factors $R_{V}, R_{T}$ and $R_{A}$, respectively. The GPD $\widetilde{E}$ does not contribute at $\xi=0$.

The handbag amplitudes (3) lead to the following result for the Compton cross section

$$
\begin{aligned}
\frac{d \sigma}{d t}=\frac{d \hat{\sigma}}{d t} & \left\{\frac{1}{2}\left[R_{V}^{2}(t)\left(1+\kappa_{T}^{2}\right)+R_{A}^{2}(t)\right]\right. \\
& \left.-\frac{u s}{s^{2}+u^{2}}\left[R_{V}^{2}(t)\left(1+\kappa_{T}^{2}\right)-R_{A}^{2}(t)\right]\right\}+O\left(\alpha_{s}\right),
\end{aligned}
$$

where $d \hat{\sigma} / d t$ is the Klein-Nishina cross section for CS of massless, point-like spin-1/2 particles of charge unity. The parameter $\kappa_{T}$ is defined as

$$
\kappa_{T}=\frac{\sqrt{-t}}{2 m} \frac{R_{T}}{R_{V}}
$$


Another interesting observable in CS is the helicity correlation, $A_{L L}$, between the initial state photon and proton or, equivalently, the helicity transfer, $K_{L L}$, from the incoming photon to the outgoing proton. In the handbag approach one obtains ${ }^{5,6}$

$$
A_{L L}=K_{L L} \simeq \frac{s^{2}-u^{2}}{s^{2}+u^{2}} \frac{R_{A}}{R_{V}}+O\left(\kappa_{T}, \alpha_{s}\right)
$$

where the factor in front of the form factors is the corresponding observable for $\gamma q \rightarrow \gamma q$. The result (7) is a robust prediction of the handbag mechanism, the magnitude of the subprocess helicity correlation is only diluted somewhat by the ratio of the form factors $R_{A}$ and $R_{V}$. On the other hand, the helicity correlation for sideways polarized protons (i.e. perpendicular to the proton's three-momentum and in the scattering plane), is very sensitive to details of the approach. It reads

$$
A_{L S}=-K_{L S} \simeq \frac{-t}{s-u} \frac{R_{A}}{R_{V}} \kappa_{T}\left[1+\frac{2 m}{\sqrt{s}} \frac{\sqrt{-t}}{\sqrt{s}+\sqrt{u}} \kappa_{T}^{-1}\right]+O\left(\alpha_{\mathrm{s}}\right) .
$$

\section{The large- $t$ behaviour of GPDs}

In oder to make actual predictions for CS however models for the soft form factors or rather for the underlying GPDs are required. A first attempt to parameterize the GPDs $H$ and $\widetilde{H}$ at zero skewness has been given in ${ }^{2,3,5}$

$$
\begin{aligned}
& H^{a}(\bar{x}, 0 ; t)=\exp \left[a^{2} t \frac{1-\bar{x}}{2 \bar{x}}\right] q_{a}(\bar{x}), \\
& \widetilde{H}^{a}(\bar{x}, 0 ; t)=\exp \left[a^{2} t \frac{1-\bar{x}}{2 \bar{x}}\right] \Delta q_{a}(\bar{x}),
\end{aligned}
$$

where $q(\bar{x})$ and $\Delta q(\bar{x})$ are the usual unpolarized and polarized parton distributions in the proton ${ }^{\mathrm{a}}$. $a$, the transverse size of the proton, is the only free parameter and even it is restricted to the range of about 0.8 to 1.2 $\mathrm{GeV}^{-1}$. Note that $a$ essentially refers to the lowest Fock states of the proton which, as phenomenological experience tells us, are rather compact. The model (9) is designed for large $-t$. Hence, forced by the Gaussian in (9), large $\bar{x}$ is implied, too. Despite of this the normalization of the model GPDs at $t=0$ is correct.

With the model GPDs (9) at hand one can evaluate the various form factors by taking appropriate moments, e.g.

$$
F_{1}=\sum_{q} e_{q} \int_{-1}^{1} d \bar{x} H^{q}(\bar{x}, 0 ; t), \quad R_{V}=\sum_{q} e_{q}^{2} \int_{-1}^{1} \frac{d \bar{x}}{\bar{x}} H^{q}(\bar{x}, 0 ; t) .
$$

a The parameterization (9) can be motivated by overlaps of light-cone wave functions which have a Gaussian $\vec{k}_{\perp}$ dependence ${ }^{2,3,7}$. 

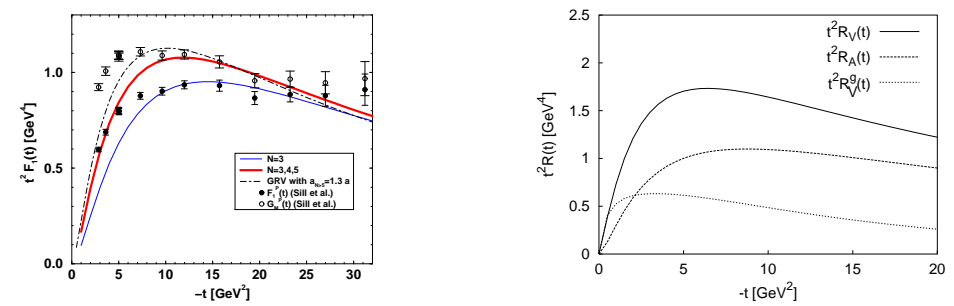

Figure 2. The Dirac form factor of the proton (left) and the Compton form factors (right). The figures are taken from Refs. ${ }^{3,5}$, data are taken from Ref. ${ }^{8}$.

Results for the form factors are shown in Fig. 2. Obviously, as the comparison with experiment ${ }^{8}$ reveals, the model GPDs work quite well in the case of the Dirac form factor. The scaled form factors $t^{2} F_{1}$ and $t^{2} R_{i}$ exhibit broad maxima which mimick dimensional counting in a range of $-t$ from, say, 5 to about $20 \mathrm{GeV}^{2}$. The position of the maximum of any of the scaled form factors is approximately located at ${ }^{6}$

$$
t_{0} \simeq-4 a^{-2}\left\langle\frac{1-\bar{x}}{\bar{x}}\right\rangle_{F(R)}^{-1} .
$$

The mildly $t$-dependent mean value $\langle(1-\bar{x}) / \bar{x}\rangle$ comes out around $1 / 2$. A change of $a$ moves the position of the maximum of the scaled form factors but leaves their magnitudes essentially unchanged.

The Pauli form factor $F_{2}$ and its Compton analogue $R_{T}$ contribute to proton helicity flip matrix elements and are related to the GPD $E$ analogously to (10). This connection suggests that, at least for not too small values of $-t, R_{T} / R_{V}$ roughly behaves as $F_{2} / F_{1}$. Thus, on the basis of the SLAC data ${ }^{9}$ on $F_{2} / F_{1}$, one expects $R_{T} / R_{V} \propto m^{2} / t$ while the recent JLab data ${ }^{10}$ rather indicate a behaviour as $\propto m / \sqrt{-t}$. If the first estimate is correct the contribution from the form factor $R_{T}$ to Compton scattering can be ignored while in the second case it is to be taken into account since it contributes to the same order in $\Lambda / \sqrt{-t}$ as the other form factors. Since it is not yet clear which behaviour is the correct one, predictions for Compton observables are given for two different scenarios. Both $R_{T}$ and $\alpha_{\mathrm{s}}$ corrections are omitted in scenario B but taken into account in A where the ratio $\kappa_{T}$ is assumed to have a value of 0.37 as estimated from the JLab form factor data ${ }^{10}$.

Employing these model GPDs and the corresponding form factors, various Compton observables can be calculated ${ }^{3,5,6}$. The predictions for the differential cross section are in fair agreement with experiment. The approximative $s^{6}$-scaling of the predictions is related to the broad maximum 

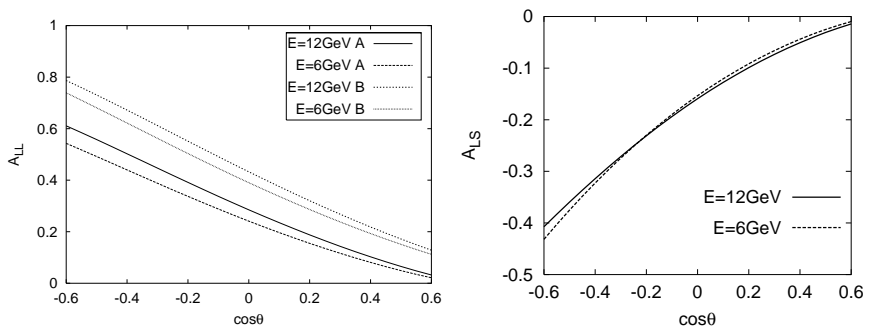

Figure 3. Predictions for the helicity correlations $A_{L L}=K_{L L}$ (left) and $A_{L S}=-K_{L S}$ (right) ${ }^{5}$. NLO corrections and the tensor form factor are taken into account (scenario A), in scenario B they are neglected.

the scaled form factors exhibit, see Fig. 2. JLab will provide accurate cross section data soon which will allow a detailed examination of the handbag mechanism. Predictions for $K_{L L}$ and $K_{L S}$ are shown in Fig. 3. The JLab E99-114 collaboration ${ }^{11}$ has presented a first measurement of $K_{L L}$ and $K_{L S}$ at a c.m.s. scattering angle of $120^{\circ}$ and a photon energy of $3.23 \mathrm{GeV}$. These still preliminary data points are in fair agreement with the predictions from the handbag given the small energy at which they are available. The kinematical requirement of the handbag mechanism $s,-t,-u \gg \Lambda^{2}$ is not well satisfied and therefore one has to be aware of large dynamical and kinematical corrections. Among them there are proton mass effects which have been investigated in Ref. ${ }^{12}$.

There is an alternative to the handbag factorization. This is the leadingtwist scheme ${ }^{13}$ where all valence quarks the involved hadrons are made up participate in the hard scattering and not just a single one. Although it is believed for good reasons that the leading-twist scheme dominates for asymptotically large $s$, it is not clear theoretically which of the two approaches provides the appropriate description of wide-angle Compton scattering at, say, $-t \simeq 10 \mathrm{GeV}^{2}$. The ultimate decision is to be made by experiment. In fact, leading-twist calculations, e.g. ${ }^{14}$, reveal difficulties with the size of the Compton cross section, the numerical results are way below experiment. Moreover, the leading-twist approach leads to a negative value for $K_{L L}$ at angles larger than $90^{\circ}$ where the handbag predictions are positive (see Fig. 3). $K_{L S}$ is zero to leading-twist order.

For large $-t$ and $\bar{x} \gtrsim 0.6$, the zero-skewness GPDs (9) can be parameterized as

$$
H^{q}(\bar{x}, 0, t) \simeq f_{q} \bar{x}(1-\bar{x})^{b_{q}} \exp \left[\frac{a^{2} t}{2} \frac{1-\bar{x}}{\bar{x}}\right],
$$

and analogously for the other ones; evolution is ignored for simplicity. The 
various form factors, see for instance (10), imply integrals over GPDs from 0 to 1 . However, for $-t \gtrsim 10 \mathrm{GeV}^{2}$, the exponential in (12) cuts off the small $\bar{x}$ region and, as can easily be checked numerically, it suffices to integrate from 0.6 to 1 . Hence, one can work out the large $-t$ behaviour of the form factors from the parameterization (12).

For very large values of $-t$, well above $100 \mathrm{GeV}^{2}$, the form factors behave as 3,15

$$
F_{1}^{q}, R_{V, A}^{q} \propto(-1 / t)^{b_{q}+1} .
$$

This correspondence between the large- $\bar{x}$ behaviour of the parton distributions and the large $-t$ behaviour of the form factors is analogous to the Drell-Yan-West relation ${ }^{16}$. The asymptotic behaviour (13) emerges very slowly; for $-t$ near $10 \mathrm{GeV}^{2}$ the form factors effectively behave $\propto t^{2}$ as can be seen from Fig. 2 .

Using $b_{u}=b_{d} \simeq 3$ and $b_{\text {sea }} \simeq 7$ in agreement with overlaps evaluated from $\mathrm{SU}(6)$ symmetric wave functions ${ }^{3,15 \mathrm{~b}}$, one sees that the active quarks in the handbag are valence quarks for large $-t$. This does not imply that the proton is only made of valence quarks; the bubble in the handbag, see Fig. 1, represents a sum over all parton configurations allowed by the conservation laws. Evaluating the form factors from (12) and the above powers $b_{q}$, one finds for the ratios of neutron over proton form factors

$$
\frac{d}{u} \rightarrow \rho=\frac{f_{d}}{f_{u}}, \quad \frac{F_{1 n}}{F_{1 p}} \rightarrow \frac{\rho+e_{d} / e_{u}}{1+e_{d} / e_{u} \rho}, \quad \frac{R_{1 n}}{R_{1 p}} \rightarrow \frac{\rho+\left(e_{d} / e_{u}\right)^{2}}{1+\left(e_{d} / e_{u}\right)^{2} \rho},
$$

which approximately hold for $-t \gtrsim 10 \mathrm{GeV}^{2}$. Since the vector form factor dominates the Compton cross section, the ratio of neutron over proton cross sections is approximatively given by

$$
d \sigma_{n} / d \sigma_{p} \rightarrow\left[\frac{\rho+\left(e_{d} / e_{u}\right)^{2}}{1+\left(e_{d} / e_{u}\right)^{2} \rho}\right]^{2} .
$$

Measurements of the parton distributions for $\bar{x} \gtrsim 0.6$ and of the neutron form factors at sufficiently large $-t$ would thus allow further tests of the handbag mechanism. It is to be stressed that the relations (14) do not rely on details of the parameterization (12), required is only a sufficiently strong suppression of contributions from the low- $\bar{x}$ region. The asymptotic behaviour (13), however, demands more. The effective range of $\bar{x}$ must shrink to unity with increasing $-t([1+c / t, 1]$ with $c>0)$.

${ }^{\mathrm{b}}$ In the phenomenological parton distributions, see e.g. Ref. ${ }^{17}, b_{d}$ is rather 4 than 3 with the consequence of suppressed $d$-quark contributions to the electromagnetic proton and neutron form factors for large $-t$. In the large- $\bar{x}$ region however, the errors in the phenomenological parton distributions are substantial. 


\section{The handbag mechanism in other wide-angle reactions}

The handbag approach has been applied to several other high-energy wideangle reactions. Thus, as shown in Ref. ${ }^{6}$, the calculation of real Compton scattering can be straightforwardly extended to virtual Compton scattering provided $Q^{2} /-t \ll 1$. The handbag approach also applies to reactions like $\gamma p \rightarrow \gamma \Delta\left(N^{*}\right)$. New GPDs, parameterizing the soft proton- $\Delta\left(N^{*}\right)$ matrix elements, occur in these reactions ${ }^{18}$. For the wide-angle region, however, such processes have not yet been calculated.

Photo- and electroproduction of mesons have also been discussed within the handbag approach ${ }^{19}$ using, as in deep virtual electroproduction ${ }^{20}$, a one-gluon exchange mechanism for the generation of the meson. The normalization of the photoproduction cross section is not yet understood. Either vector meson dominance contributions are still predominant or the generation of the meson by the exchange of a hard gluon underestimates the handbag contribution. Despite of this the handbag contribution to photoand electroproduction has several interesting properties which perhaps survive an improvement of the approach. For instance, the helicity correlation $\hat{A}_{L L}$ for the subprocess $\gamma q \rightarrow \pi q$ is the same as for $\gamma q \rightarrow \gamma q$, see (7). $A_{L L}$ for the full process is diluted by form factors similar to the case of Compton scattering. Another interesting result is the ratio of the cross sections for the photoproduction of $\pi^{+}$and $\pi^{-}$which is approximately given by

$$
\frac{d \sigma\left(\gamma n \rightarrow \pi^{-} p\right)}{d \sigma\left(\gamma p \rightarrow \pi^{+} n\right)} \simeq\left[\frac{e_{d} u+e_{u} s}{e_{u} u+e_{d} s}\right]^{2} .
$$

The form factors which, for a given flavor, are the same as those appearing in CS, cancel in the ratio. The prediction (16) is in fair agreement with a recent JLab measurement ${ }^{21}$ which, at $90^{\circ}$, provides values of $1.73 \pm 0.15$ and $1.70 \pm 0.20$ for the ratio at beam energies of 4.158 and $5.536 \mathrm{GeV}$, respectively.

Elastic hadron-hadron scattering can be treated as well. Details have not yet been worked out but it has been shown that form factors of the type discussed in Sect. 3 control elastic scattering, too ${ }^{6}$. The experimentally

observed scaling behaviour of these cross sections can be attributed to the broad maxima the scaled form factors show, see Fig. 2 and Eq. (11).

Two-photon annihilations into pairs of hadrons can also be calculated, the arguments for handbag factorization hold as well as has recently been shown in Ref. ${ }^{22}$ (see also Ref. ${ }^{23}$ ). The cross section for the production of a pair of pseudoscalar mesons or baryons read

$$
\frac{d \sigma}{d t}(\gamma \gamma \rightarrow M \bar{M})=\frac{8 \pi \alpha_{\mathrm{elm}}^{2}}{s^{2} \sin ^{4} \theta}\left|R_{M \bar{M}}(s)\right|^{2}
$$




$$
\begin{aligned}
\frac{d \sigma}{d t}(\gamma \gamma \rightarrow B \bar{B}) & =\frac{4 \pi \alpha_{\mathrm{elm}}^{2}}{s^{2} \sin ^{2} \theta}\left\{\left|R_{A}^{B}(s)+R_{P}^{B}(s)\right|^{2}\right. \\
& \left.+\cos ^{2} \theta\left|R_{V}^{B}(s)\right|^{2}+\frac{s}{4 m^{2}}\left|R_{P}^{B}(s)\right|^{2}\right\} .
\end{aligned}
$$

In analogy to Eq. (10) the form factors represent integrated two-hadron distribution amplitudes which are time-like versions of GPDs. The angle dependencies are in fair agreement with experiment.

A characterisic feature of the handbag mechanism in the time-like region is the intermediate $q \bar{q}$ state implying the absence of isospin-two components in the final state. A consequence of this property is

$$
\frac{d \sigma}{d t}\left(\gamma \gamma \rightarrow \pi^{0} \pi^{0}\right)=\frac{d \sigma}{d t}\left(\gamma \gamma \rightarrow \pi^{+} \pi^{-}\right)
$$

which is independent of the soft physics input and is, in so far, a robust prediction of the handbag approach. The absence of the isospin-two components combined with flavor symmetry allows one to calculate the cross sections for other $B \bar{B}$ channels using the form factors for $p \bar{p}$ as the only soft physics input.

\section{Summary}

I have reviewed the theoretical activities on applications of the handbag mechanism to wide-angle scattering. There are many interesting predictions, some are in fair agreement with experiment, others still awaiting their experimental examination. It seems that the handbag mechanism plays an important role in wide-angle exclusive reactions for momentum transfers of the order of $10 \mathrm{GeV}^{2}$. However, before we can draw firm conclusions more experimental tests are needed. The leading-twist approach, on the other hand, typically provides cross sections which are way below experiment. As is well-known the cross section data for many hard exclusive processes exhibit approximate dimensional counting rule behaviour. Infering from this fact the dominance of the leading-twist contribution is premature. The handbag mechanism can explain this approximate power law behaviour (and often the magnitude of the cross sections), too. It is attributed to the broad maxima the scaled form factors show and, hence, reflects the the transverse size of the lowest Fock states of the involved hadrons.

I finally emphasize that the structure of the handbag amplitude, namely its representation as a product of perturbatively calculable hard scattering amplitudes and $t$-dependent form factors is the essential result. Refuting the handbag approach necessitates experimental evidence against this factorization. 


\section{References}

1. A. V. Radyushkin, Phys. Rev. D56 5524 (1997) [hep-ph/9704207]; X. Ji, Phys. Rev. D55 7114 (1997) [hep-ph/9609381].

2. A. V. Radyushkin, Phys. Rev. D 58, 114008 (1998) [hep-ph/9803316].

3. M. Diehl, T. Feldmann, R. Jakob and P. Kroll, Eur. Phys. J. C 8, 409 (1999) [hep-ph/9811253].

4. M. Diehl, Eur. Phys. J. C 19, 485 (2001) [hep-ph/0101335].

5. H. W. Huang, P. Kroll and T. Morii, Eur. Phys. J. C 23, 301 (2002) [hep$\mathrm{ph} / 0110208]$.

6. M. Diehl, T. Feldmann, R. Jakob and P. Kroll, Phys. Lett. B 460, 204 (1999) [hep-ph/9903268].

7. M. Diehl, T. Feldmann, R. Jakob and P. Kroll, Nucl. Phys. B 596, 33 (2001) [Erratum-ibid. B 605, 647 (2001)] [hep-ph/0009255].

8. A.F. Sill et al., Phys. Rev. D48, 29 (1993); A. Lung et al., Phys. Rev. Lett. 70, 718 (1993).

9. L. Andivahis et al., Phys. Rev. D 50, 5491 (1994).

10. O. Gayou et al. [Jefferson Lab Hall A Collaboration], Phys. Rev. Lett. 88, 092301 (2002) [nucl-ex/0111010].

11. E99-114 JLab collaboration, spokespersons C. Hyde-Wright, A. Nathan and B. Wojtsekhowski.

12. M. Diehl, T. Feldmann, H.W. Huang and P. Kroll, hep-ph/0212138, to be published in Phys. Rev. D.

13. G. P. Lepage and S. J. Brodsky, Phys. Rev. D 22, 2157 (1980).

14. T. C. Brooks and L. Dixon, Phys. Rev. D 62, 114021 (2000) [hep$\mathrm{ph} / 0004143]$.

15. J. Bolz and P. Kroll, Z. Phys. A 356, 327 (1996) [hep-ph/9603289].

16. S. D. Drell and T. M. Yan, Phys. Rev. Lett. 24, 181 (1970); G. B. West, Phys. Rev. Lett. 24, 1206 (1970).

17. M. Gluck, E. Reya and A. Vogt, Eur. Phys. J. C 5, 461 (1998) [hepph/9806404].

18. L. L. Frankfurt, . V. Polyakov, M. Strikman and M. Vanderhaeghen, Phys. Rev. Lett. 84, 2589 (2000) [hep-ph/9911381].

19. H. W. Huang and P. Kroll, Eur. Phys. J. C 17, 423 (2000) [hep-ph/0005318].

20. J.C. Collins, L. Frankfurt and M. Strikman, Phys. Rev. D56, 2982 (1997) [hep-ph/9611433]; A. V. Radyushkin, Phys. Lett. B 385, 333 (1996) [hep$\mathrm{ph} / 9605431]$.

21. L. Y. Zhu et al. [Jefferson Lab Hall A Collaboration], nucl-ex/0211009.

22. M. Diehl, P. Kroll and C. Vogt, Phys. Lett. B 532, 99 (2002) [hep$\mathrm{ph} / 0112274]$; hep-ph/0206288.

23. A. Freund, A. V. Radyushkin, A. Schafer and C. Weiss, hep-ph/0208061; F. E. Close and Q. Zhao, Phys. Lett. B 553, 211 (2003) [hep-ph/0210277]. 